\title{
Frontières
}

\section{LAFONTAINE, Céline, La société postmortelle, Paris, Seuil, 2008, 256 p.}

\section{Sébastien St-Onge}

Volume 22, numéro 1-2, automne-printemps 2009-2010

\section{Résilience et deuil}

URI : https://id.erudit.org/iderudit/045043ar

DOI : https://doi.org/10.7202/045043ar

Aller au sommaire du numéro

\section{Éditeur(s)}

Université du Québec à Montréal

ISSN

1180-3479 (imprimé)

1916-0976 (numérique)

Découvrir la revue

Citer ce compte rendu

St-Onge, S. (2009). Compte rendu de [LAFONTAINE, Céline, La société postmortelle, Paris, Seuil, 2008, 256 p.] Frontières, 22(1-2), 133-134.

https://doi.org/10.7202/045043ar

Ce document est protégé par la loi sur le droit d'auteur. L'utilisation des services d'Érudit (y compris la reproduction) est assujettie à sa politique d'utilisation que vous pouvez consulter en ligne.

https://apropos.erudit.org/fr/usagers/politique-dutilisation/
Cet article est diffusé et préservé par Érudit.

Érudit est un consortium interuniversitaire sans but lucratif composé de l'Université de Montréal, l'Université Laval et l'Université du Québec à Montréal. Il a pour mission la promotion et la valorisation de la recherche. https://www.erudit.org/fr/ 


\section{LAFONTAINE, Céline \\ La société postmortelle}

Paris, Seuil, 2008, 256 p.

\section{Céline Lafontaine}

\section{SOLELTPST
MORTELLE}

SEUIL 㗈

La mort est devenue, ces dernières décennies, un objet prisé pour étudier la transformation du lien social. Nombreux sont les praticiens des sciences humaines qui ont postulé que la mort était à l'origine de toute culture. Dans une formule maintenant consacrée, Louis-Vincent Thomas a soutenu que "toute société se voudrait immortelle et que ce qu'on appelle culture n'est rien d'autre qu'un ensemble organisé de croyances et de rites, afin de lutter contre le pouvoir dissolvant de la mort individuelle et collective » (Mort et pouvoir, Payot, 1978, p. 10). À partir de ce postulat fondateur, comment la société moderne, celle-là même qui a substitué la technologie à l'eschatologie et qui a décomposé l'unité de la mort en une multitude de causes, arrive-t-elle à lutter contre la finitude? Comment, au fond, la «mort-absolue», celle qui prévalait depuis l'aube des temps, est-elle devenue une maladie que I'arsenal biotechnologique a désormais le mandat de combattre, voire de vaincre?

Dans La société postmortelle, Céline Lafontaine répond à ces questions fondamentales en démontrant que les avancées biomédicales des deux derniers siècles ont brouillé considérablement les frontières entourant le mourir et la mort. La sociologue de I'Université de Montréal amène le lecteur à arpenter ces frontières ténues et à cadastrer avec elle les nouveaux territoires sociaux, médicaux et juridiques que la société postmortelle tend à induire. Au-delà du titre accrocheur de l'ouvrage, le lecteur se demandera, très tôt dans sa lecture, à quoi renvoie exactement la société postmortelle? Ficelée à la théorie sociologique générale de Michel Freitag, la postmortalité, nous avise l'auteure, doit être située dans un cadre théorique plus large, celui de la postmodernité, qui se fonde sur un nouveau mode de régulation sociale axé sur la gestion informationnelle et l'opérationnalité technoscientifique. Voulant marquer le nouveau rapport que les contemporains entretiennent à l'égard de la mort, "[l]a notion de postmortalité renvoie plus spécifiquement à la volonté affichée de vaincre techniquement la mort, de "vivre sans vieillir" et de prolonger indéfiniment la vie» (p. 14).

L'ouvrage comporte six chapitres qui explorent le double processus de déconstruction et de désymbolisation de la mort. Toutefois, la presque totalité de l'essai est consacrée à la déconstruction technoscientifique et biomédicale de la mort. Ce déséquilibre est largement contrebalancé par la richesse interprétative du premier pôle étudié. Pas à pas, cette genèse de la déconstruction scientifique de la mort amène le lecteur à constater comment, si l'on emprunte une image à l'organicisme, le corps social contemporain en est venu à déployer des anticorps qui sont propres à son nouveau mode de reproduction sociale. $\mathrm{Ne}$ serait-ce qu'à ce seul titre, l'ouvrage de Lafontaine jette un éclairage plus que pertinent sur les mécanismes de défense que la société postmoderne a sécrétés pour se défendre contre la force destructrice de la mort. Reste à savoir, et cette interrogation traverse le livre d'un couvert à l'autre, si ces nouveaux mécanismes de défense, plus structurels que symboliques, aident réellement les contemporains à mieux vivre avec la conscience prospective de la mort. Autrement dit, la technoscience, qui culmine avec l'avènement des nanotechnologies, est-elle plus efficiente pour effectuer l'espacement fondamental entre le monde des vivants et celui des morts? Chose certaine, cette substitution dans les mécanismes de défense du corps social marque une rupture historique sans précédent dans I'histoire de I'Occident. Car, comme nous le rappelle la sociologue, en ramenant le lecteur en arrière de sa société, du Moyen Âge au début de la période moderne, «le refus chrétien de la mort est entièrement tourné vers la promesse d'une vie éternelle incarnée par la figure du Christ» (p. 27). À l'angoisse de la mort, le christianisme répond par l'immortalité de l'âme individuelle. L'eschatologie chrétienne demeure le principal ressort culturel dont dispose la société traditionnelle pour reprendre, à l'avantage de la vie et de l'ensemble du corps social, le nonsens de la mort.

Mais voilà que le siècle des Lumières change la donne. "L'instant fatidique personnifié par la Grande Faucheuse, souligne Lafontaine, se dissout peu à peu pour faire place à une conception de la mort en termes de phénomène biologique» ( $p .71)$. Certes, on continue de mourir, mais la mort n'émane plus d'un dehors obéissant à la volonté divine; elle est dorénavant un phénomène naturel qui laisse prise à l'investigation médicale, ne serait-ce que dans la recension de ses principaux traits physionomiques. Naturalisée, la mort «[...] prend le visage de la maladie, dont elle n'est plus que le terme final » (p. 35). II n'en faut pas davantage pour que la modernité déclare officiellement la guerre à sa plus grande menace. Dans cet esprit, la finitude devient un fait insensé qui interrompt à la fois l'ennoblissement de l'être et de la société tout entière. «Parce que [la mort] n'est pas une punition, parce qu'elle n'est pas une fatalité mais une loi naturelle, il est non seulement légitime mais raisonnable de s'en défendre, de tenter d'en reculer la date " (Michel Vovelle, La mort et l'Occident, 2000, cité par Lafontaine, p. 29).

La science anatomico-clinique du début du XIX'e siècle et la science médicale du $X X^{e}$ siècle ne font que poursuivre le procès amorcé par les Lumières en ouvrant la boîte de Pandore des causes infinies de la mort. L'époque où il suffisait de placer un miroir devant la bouche du mourant, afin de vérifier s'il respirait encore, apparaît révolue. Les étapes physiologiques menant au constat du décès modulent en fonction des avancées technologiques et ces constructions médicales altèrent les frontières entre la vie et la mort. Suffit-il que le cœur, à titre d'exemple, cesse de battre pour certifier la mort d'un individu? Et que faire quand le respirateur artificiel maintient en vie celui qui, sans la machine incarnant le progrès technique, trouverait assurément la mort? Où loge donc la mort? «Paradoxalement, remarque Lafontaine, plus la science médicale se développe, plus la possibilité d'intervenir dans le processus final augmente, moins il devient possible d'identifier clairement le moment précis du décès» (p. 73). La découverte de l'électroencéphalogramme, au terme des années 1950, force les instances médicales et étatiques à clarifier le statut juridique de la mort. À la faveur du don d'organes, le siège de la vie se déplace du système cardiovasculaire au cerveau.

Les avancées technoscientifiques ne s'arrêteront pas à l'électroencéphalogramme. Depuis la Deuxième Guerre mondiale, les tenants de la cybernétique pensent le corps humain en termes de système. Si le corps est un système entropique, c'est-à-dire qu'il tend naturellement vers un état de dégénérescence, et que l'information en est la clé, il importe maintenant de déchiffrer son réseau informationnel, et ce, dans le but éventuel de retarder la détérioration du systèmecorps. Couplées à la cybernétique, la biologie moléculaire et la culture des cellules souches apparaissent rapidement pour certains scientifiques comme une arme pouvant retarder la sénescence entropique. "À l'image ancienne de la mort comme une faucheuse surgissant du dehors pour détruire s'est surimposée, au niveau cellulaire tout au moins, une image radicalement nouvelle, celle d'un sculpteur, au cœur du vivant, à l'œuvre dans l'émergence de sa forme et de sa complexité " (JeanClaude Ameisen, "L'oubli de nos métamorphoses. Aperçus sur les mécanismes d'autodestruction cellulaire », dans La mort et I'Immortalité, 
Bayard, 2004, cité par Lafontaine, p. 118). S'inspirant de la salamandre, la médecine régénérative développe "une série de stratégies et d'interventions thérapeutiques visant à réparer ou à remplacer les tissus endommagés du corps humain, à la suite d'accidents ou de maladies dégénératives» (p. 124). En cherchant à retarder l'entropie du corps, la médecine régénérative vient altérer la distinction entre normal et pathologique, entre vieillissement et maladie. «Il ne s'agit donc plus, écrit la sociologue de Montréal, comme pour la médecine clinique, de conserver l'état d'équilibre du corps en luttant contre les maladies, mais plutôt de combattre la dégénérescence en tant que telle » (p. 146). Récemment, les nanotechnologies ont relancé les rêves les plus utopiques des posthumanistes. Issues de la convergence entre plusieurs domaines de recherche - physique quantique, informatique, biologie moléculaire et microélectronique--, les nanotechnologies portent plus que jamais l'espoir de repousser la mort dans ses derniers retranchements.

Du respirateur artificiel aux nanotechnologies en passant par l'électroencéphalogramme, les avancées technoscientifiques des deux derniers siècles vont favoriser, constate Lafontaine, l'émergence d'un biopouvoir dont l'État sera le principal instrument. À la suite du plan Beveridge, en 1942, la responsabilité des États nationaux ne se limitera plus à la sécurité des citoyens, mais elle en comportera une seconde: la santé. "Qu'il soit question des normes d'hygiène publique, des réglementations sécuritaires, des multiples prescriptions alimentaires et sportives formulées par les pouvoirs publics, la volonté de "tuer la mort" est indéniablement l'enjeu principal du biopouvoir» (p. 39). Concrètement, les individus, désormais conscientisés à la dangerosité de vivre, s'en remettent aux experts spécialistes pour identifier les principaux facteurs à éviter pour repousser la mort: tabagisme, obésité, restriction calorifique, viande rouge, cholestérol et sédentarité en sont les principales illustrations.

Mais ce biopouvoir ne peut se maintenir en place sans une transformation profonde de la culture. Au détriment du lien social, s'inquiète la sociologue de Montréal, la vie en elle-même sera sacralisée jusqu'à renverser l'idéal des Lumières. «Alors [qu'il] reposait sur la croyance en la perfectibilité de la société, sur la volonté d'améliorer les conditions de vie par le biais de l'action collective, la société postmortelle se caractérise par la croyance en la perfectibilité de la vie elle-même» (p. 121). Cette biologisation de la culture s'alimente au fantasme de l'amortalité, fantasme qui n'est pas sans rompre lui aussi avec I'histoire de l'Occident. II ne s'agit plus "d'accéder à un autre monde, ni même d'échapper complètement à la condition humaine, mais de poursuivre éternellement la vie ici-bas» (p. 156). Dans ce contexte, la vieillesse devient paradoxalement une pathologie puisque ses traits annoncent ce que la science n'est pas encore parvenue à éradiquer: la mort. Chargée de vaincre la mort, la science doit s'acquitter d'une seconde tâche, celle d'estomper les ravages du temps.

Le biopouvoir et la biologisation de la culture, ces deux faces de la postmortalité, ont été possibles dans la mesure où les modernes, grâce au recul de la mortalité infantile au $X X$ siècle et à l'augmentation considérable de l'espérance de vie, ont pu se projeter dans l'avenir. Ces deux phénomènes ont amené, selon l'expression de Marcel Gauchet, "une redéfinition des âges de la vie » qui n'est pas sans porter préjudice à l'équilibre filial sur lequel reposait le cycle de la vie. S'amalgamant parfaitement à l'individualisme libéral, le droit à la vie, voire à prolonger la vie, risque, c'est du moins le danger que soulève Lafontaine, de se substituer à la filiation. Les babyboomers, bénéficiaires des progrès technoscientifiques et principaux ambassadeurs des espoirs de la société postmortelle, ne risquentils pas de repousser encore plus loin la frontière départageant les soins nécessaires et ceux de performance? Considérant leur poids démographique et politique, eux qui s'engagent dans les territoires avancés de l'âge, succomberont-ils à la tentation de rediriger les sommes allouées à la santé aux fins de leur longévité et au détriment de la génération montante? Chose certaine, "[l]'accès aux soins de santé et aux techniques biomédicales de pointe sera vraisemblablement l'un des enjeux sociopolitiques les plus sensibles des prochaines décennies » (p. 223-224).

Dans son dernier chapitre, et à la toute fin de son itinéraire, la sociologue aborde brièvement la désymbolisation de la mort. Elle utilise la transformation récente des rituels funéraires, la montée de la crémation et les débats entourant l'euthanasie et le suicide assisté pour illustrer la désocialisation qui marque le rapport des contemporains à la mort. Si, au ras de l'existence, la mort continue de sévir, "ce qui s'efface en revanche, écrit-elle, c'est son statut ontologique, c'est-à- dire son rôle fondamental dans l'édification de la culture, de l'ordre symbolique, qui donne sens à l'existence et au monde » (p. 187). S'étant libéré des principaux liens institutionnels qui le reliaient à la société, l'individu de la société postmortelle, dont l'autonomie subjective est le principal moteur, serait en train de déverrouiller les derniers écrous normatifs le reliant à la société. S'il est vrai que le procès moderne va en ce sens, il n'en demeure pas moins que les funérailles et le suicide assisté nécessitent encore le détour obligé de l'autre. Bien que l'ouvrage de Lafontaine boude I'héritage des études québécoises sur la mort, il n'en demeure pas moins pertinent pour autant.

Sébastien St-Onge,

professeur de sociologie,

Collège Lionel-Groulx.

\section{WILSON, Tim \\ Griefwalker - \\ L'accompagnateur}

DVD-vidéo, son, coul., Office national du film, 2008, $70 \mathrm{~min} .09 \mathrm{~s}$.

Dans ce documentaire, le réalisateur Tim Wilson dresse un portrait

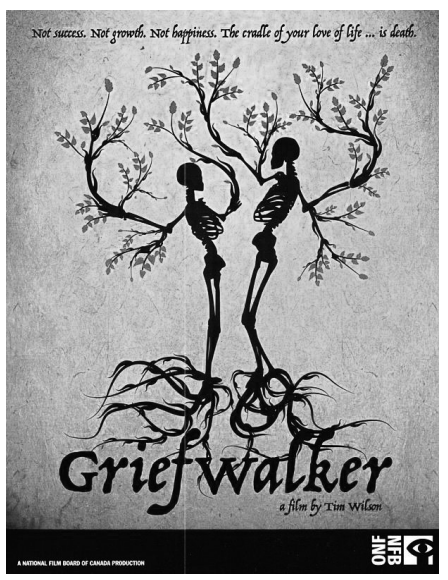

de son ami de longue date, Stephen Jenkinson, qui l'a accompagné, il y a quelques années, alors qu'il luttait avec le déni de la mort au cours d'une maladie grave.

Stephen Jenkinson est un "activiste spirituel», conseiller en soins palliatifs et accompagnateur des mourants à l'Hôpital de Mount Sinai à Toronto. II détient une maîtrise en travail social de l'Université de Toronto ainsi qu'une maîtrise en théologie de I'Université Harvard.

Tout au long du film, nous suivons Jenkinson dans ses contacts avec des personnes confrontées à la mort. La scène dans laquelle il encourage des parents à ramener à la maison leur petite fille mourante est particulièrement émouvante.
On le retrouve également enseignant à des cliniciens, les encourageant à dire "les vraies choses" aux patients et à leur famille car, selon lui, la vérité les aide plus dans leur cheminement que le non-dit dont ils se doutent. Jenkinson transmet un message qui n'est guère apprécié dans notre société: que la mort ne doit pas être niée ou évitée mais apprivoisée. "Dans notre culture, on nous impose de ne pas savoir qu'on va mourir. Quand vous apprenez que vous allez mourir, ne mettez pas cette nouvelle de côté, gardez-la comme un bien précieux, ce qu'elle est, en fait. »

Souvent appelé l'Ange de la mort, Jenkinson accepte d'être le témoin fidèle de la difficulté de mourir. II assume pleinement son rôle de témoin de l'anxiété et de la dépression vécues par les malades en fin de vie. Il ose aborder la peur, voire la "terreur» de la mort - discours novateur que I'on a besoin d'entendre dans la pratique des soins palliatifs, et insiste sur l'importance du chagrin que l'approche de la mort provoque. "Le chagrin, dit-il, est le réveil, un signe que la vie bouge en elle-même - intégrer le chagrin et la mort dans la vie. »

Wilson dresse un portrait saisissant d'un personnage provocateur et admet que son documentaire agit comme un lent détonateur qui nous fait prendre conscience que «le creuset qui fait de nous des êtres humains c'est la mort. Ce n'est pas le succès, ni la réussite, ni le bonheur, c'est la mort. C'est la mort qui nous fait aimer la vie."

Si tu dois apprendre ta propre mort par l'entremise de quelqu'un, tu peux te poser la question: jusqu'à quel point es-tu en contact avec ta propre vie? C'est une question que les gens qui ne sont pas au seuil de la mort devraient porter en eux. Comment es-tu affecté à chaque seconde de ta vie par le fait de savoir que tu vas mourir.

Ce film, qui ne peut laisser indifférent, est aussi un beau voyage méditatif, avec de fort belles images évoquant l'impermanence de la nature - nature dans laquelle Jenkinson trouve sa force en pratiquant certaines coutumes de la culture traditionnelle autochtone, ce qui est peu banal pour un théologien de Harvard!

Il est possible de se procurer le DVD de ce documentaire en version originale avec sous-titres français en communiquant avec <contact@ orphanwisdom.com>.

Claude Cornillon-Richard Maîtrise en sciences des religions 